\title{
When does perceived lightness depend on perceived spatial arrangement?
}

\author{
ALAN L. GILCHRIST \\ State University of New York, Stony Brook, New York 11790
}

\begin{abstract}
Experiments have recently been reported in which a decisive change in perceived lightness was produced by a change in perceived spatial position, with no important change in the retinal image. A number of previous studies had found little or no such effect. Experiments of the kind that produced these effects and of the kind that do not produce these effects are presented here. The main differences between these two kinds of experiments are discussed. One difference is whether the display allows the target to be part of one ratio in one spatial position but another in the other spatial position. Another difference concerns the range of luminances within the display. Also discussed are the implications of these findings for cognitive vs. S-R theories, the order of processing depth and lightness, laboratory data vs. experience, the role of lateral inhibition in lightness perception, and theories of lightness perception in general.
\end{abstract}

The percentage of light a surface reflects is called the reflectance (or albedo) of the surface. The phenomenal counterpart of reflectance is called lightness. The simplest and most obvious determinant of lightness would seem to be the absolute amount of light (luminance) reflected by a surface, as measured by the eye. This account immediately fails because the amount (as opposed to percentage) of light a surface reflects is determined as much by the amount of light striking the surface as by the reflectance of the surface. The remarkable fact is that we perceive surface reflectance with rough accuracy despite wide variations in illumination. These facts produce what has been called the constancy question, that is, how the apparent lightness of surfaces remains so constant when variations in the amount of illumination create great variations in the absolute amount of light that these surfaces reflect. An additional question, made relevant by theoretical differences, concerns what role, if any, processes of depth perception play in lightness constancy. Consider how the major theories of lightness perception answer these questions.

For many years, the conventional explanation of lightness constancy was that of Helmholtz (1867/1962), who maintained that the level of illumination is unconsciously taken into account in evaluating the intensity of the reflected light. Such a theory makes intuitive sense since we do seem to be aware of different levels of illumination, both over time and within

This research was conducted at the Institute for Cognitive Studies, Rutgers University, Newark, New Jersey, as part of a doctoral dissertation. I wish to thank $H$. Wallach, D. Dinnerstein, and C. H. Harris for their contributions to this work. I must also mention my deep indebtedness to Irvin Rock for the outstanding training and advice I have received from him. a single scene. Moreover, depth perception plays a central role in this theory since an assessment of the various levels of illumination within a given scene would seem to depend upon information about the three-dimensional layout of the environment. For example, spatial factors such as the distance from the light source to the surface and the slant of the surface with respect to the light source would be essential to an accurate evaluation of illumination, and these factors require good depth information.

However, Hering (1874/1964) pointed out a logical problem that has since come to be known as Hering's paradox. Since both surface reflectance and illum:nation affect the observer via a single variablenamely, the intensity of reflected light - the observer would need to know the reflectance of a surface to correctly deduce the amount of illumination falling thereon. But the correct perception of surface reflectance is precisely what Helmholtz' theory attempts to account for. No one has explained how the Helmholtzian formula would escape this circularity.

In 1948, Hans Wallach suggested that the correlate of perceived lightness is to be found in ratios of luminance, not absolute luminances. He supported this argument with his now-classic experiment showing that disks of quite different luminance appear equal in lightness when their disk/background luminance ratios are equal. In addition to the striking results of the laboratory experiment, Wallach's idea gained force by the observation that when the general level of illumination in the environment changes, it is precisely the luminance ratios that remain constant. Thus, it appeared Wallach had solved Hering's paradox. The appeal to perceived illumination was rendered unnecessary. In fact, it seemed that even depth 
perception could be regarded as irrelevant to lightness perception. The perceived lightness of a given surface is held to depend simply upon the ratio of the luminance of the target surface to the luminance of the surface (or surfaces) that abuts the targets in the retinal image, regardless of where those surfaces are perceived to lie in three-dimensional space.

Partly because of Wallach's emphasis on retinally adjacent intensities, many researchers have concluded that lateral inhibition provides the neural mechanism underlying these ratio effects. This mechanism makes the output of cells dependent not only upon their own level of stimulation, but also upon the level of stimulation of adjacent cells.

Widely accepted theories of lightness perception in which lateral inhibition plays a key role have been put forth by both Cornsweet (1970) and Jameson and Hurvich (1964). While there are important conceptual differences among the theories of Cornsweet, Jameson and Hurvich, and Wallach, they can be grouped together for purposes of this paper. That is because they all hold perceived lightness to be determined by the retinal pattern. Thus, any finding that perceived lightness depends to any large extent on perceived spatial position (with the retinal array held constant) would pose serious problems for all of these theories.

In fact, the belief that lightness perception could not be completely explained at the retinal level has led to a number of studies aimed at uncovering the role of depth in lightness perception. The general plan of these studies has been to produce a change in the apparent location of a target surface without allowing any change in the pattern of light (and hence the ratios) reaching the eye.

Hochberg and Beck (1954) placed a trapezoidalshaped card vertically on a table, directly below an overhead lightbulb. Viewed monocularly from a unique viewpoint, the card appeared to be rectangular and lying flat on the table. Nine out of ten observers saw the card as darker gray when it appeared horizontal than when it appeared vertical. Although Hochberg and Beck did not report the magnitude of the effect, later replications by Beck (1965) and Flock and Freedberg (1970) found it to be on the order of about half a Munsell step. Epstein (1961) replicated the study and found no effect. However, in a related experiment, using a more complicated array, Beck (1965) obtained an effect of 1.2 Munsell steps.

A second approach has been to produce, by means of stereoscopic cues, a separation in depth between the target and its background. Gibbs and Lawson (1974) and Julesz (1971), using the classic simultaneous brightness contrast display, caused the two gray squares to appear suspended in midair, out in front of the white and black backgrounds, the retinal projection remaining essentially constant. They found that this produced no change in the strength of the contrast effect.

On the other hand, Gogel and Mershon (1969), using a Gelb-effect paradigm, compared the darkening effect of a (small) white disk placed at the center of, and coplanar with, the larger Gelb disk, with the darkening effect of the same white disk when it appeared closer to the observers in stereoscopic space. They obtained a difference of .6 of a Munsell step between matches made to the Gelb disk in the two conditions. Mershon (1972), in a closely related experiment, produced an effect of $1 \frac{11 / 4}{4}$ Munsell steps.

While some of these studies found an influence of depth perception, the effect has been one of damning the importance of depth perception with faint praise. Many have come to regard these findings as indicating that cognitive factors such as apparent spatial position can exert only a small "tuning" effect on the basic retinally produced neural signals.

Recently (Gilchrist, 1977), I presented the results of experiments in which apparent spatial position had a decisive effect on perceived lightness. In fact, under some conditions it was found that a change merely in apparent spatial position (with no change in the retinal projection) can produce a change in perceived lightness of 5.5 Munsell steps, essentially from black to white or white to black. Those experiments were part of a larger series of experiments, some of which found no influence of depth on lightness perception. How did the experiments showing strong depth effects differ from those showing none? And how did the experiments showing strong depth effects differ from the previous studies by other investigators showing little or no effect of depth on lightness? These questions will be answered in this paper.

The experiments reported here can be grouped into four parts. All involved a depth illusion. That is, a target surface was made to appear in either of two separate planes. In the first three parts, the separate planes were perpendicular to each other, while in the fourth part, the planes were parallel but separated in distance from the observer. Part 1 involved conditions in which a change in depth does not produce a change in lightness. Part 2 was analogous to Part 1 except that conditions were introduced that allowed lightness to change as a result of changes in apparent spatial position. Part 3 consisted of a critical test between a retinal-ratio conception and a planaritydependent ratio conception. Part 4 simply extended the findings to the case of parallel planes. In addition, however, the depth cue used to produce the depth illusion in Part 4 was interposition, while in Parts 1 to 3 , the effective depth cue was stereopsis. 
Parts 3 and 4, incidentally, were presented in the earlier report of this work (Gilchrist, 1977), but are described here in detail.

\section{PART 1 Perpendicular Planes, No Depth Effect}

\section{Method}

Apparatus. The laboratory arrangements are shown in Figure 1. The stimulus consisted of two perpendicular surfaces, joined along a common edge. One was a $31 / 4-i n$. horizontal square, which will henceforth be referred to as the upper target; the other, referred to as the lower target, was a vertical trapezoid, $31 / 4$ in. in height and 4 in. along its base. When viewed monocularly from a critical viewpoint, the trapezoid projected the same retinal shape as would have been produced by a second $31 / 4$-in. square lying horizontally in front of the first and coplanar with it. The stimulus was supported by, and prevented the observer's view of, a wire frame, the tip of which extended 20 in. from the background to which it was attached. The background stood at a 45-deg angle to the floor and measured 38 in. from lower front to upper back and 25 in. in width. It was covered with a light gray paper that had a reflectance of $60 \%$ and a measured luminance of $17 \mathrm{fl}$.
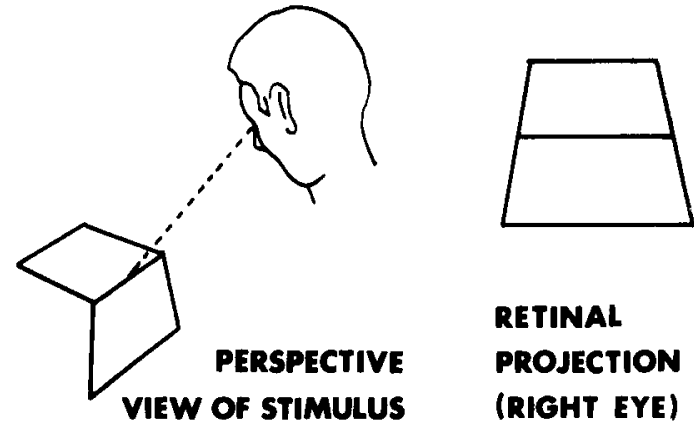

(RIGHT EYE)

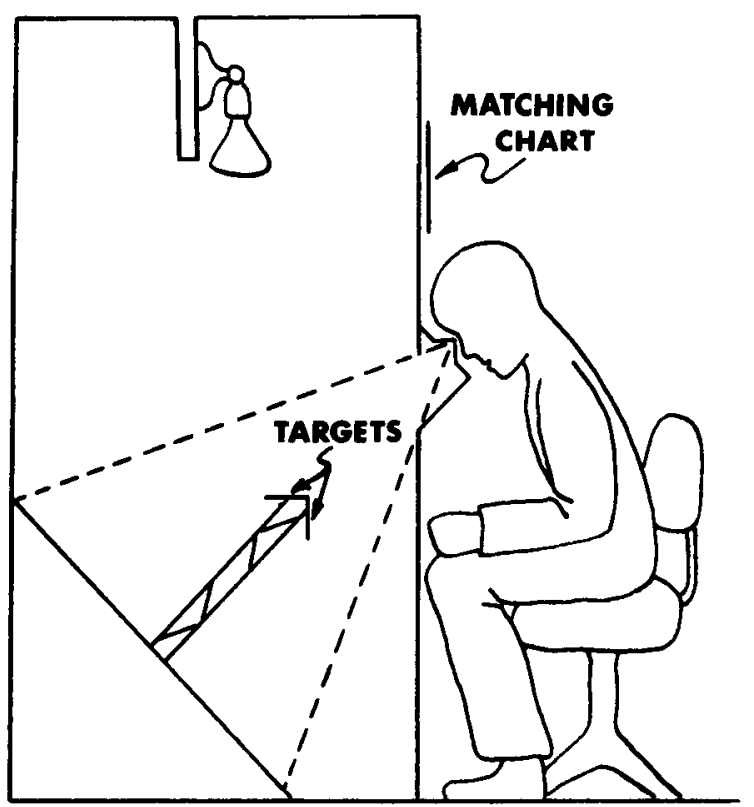

Figure 1. Arrangements used in perpendicular planes experiments.
The display was illuminated by a 150-W floodlight bulb, unseen by the observer, placed $31 \frac{1 / 2}{2}$ in. above the upper target and 6 in. behind the vertical target. The lower target was primarily illuminated by light reflected from a piece of white paper atiached to the vertical screen directly opposite the lower target. The size of this piece of paper was adjusted so that the amount of light it reflected onto the lower target was such as to make a white lower target equal in luminance to a black upper target. Thus, the upper target received 30 times as much light as the lower target, which was in relative shadow.

The observer at behind the vertical screen and viewed the stimulus by looking 45 deg downward through two conical eyepieces, each of which contained an aperture $3 / 16$ in. in diameter. Baffles in the sereen, just ahead of the eyepieces, restricted the ohserver's visual angle to approximately $47 \mathrm{deg}$ vertically and $35 \mathrm{deg}$ horizontally. The left eyepiece was adjustable left and right in order 10 match the interocular distance of each observer and could be covered by a shutter for monocular viewing.

A 16-step Muncell chart of achromatic colors, on which 2 was the darkest black and 9.5 was the lightest white, was located on the outside of the ucreen just above the observer's head. It was illuminated by a $1(x)-W$ light bulb located just above and behind the observer's head, so that the luminances of the 9.5 , the 5 (medium gray), and the 2 were $26.2,5.8$, and $.97 \mathrm{fL}$, respectively.

Design. Four utimulus displays were used (see Figure 2). In Displays 1, 2, and 3, the upper and lower targets had the same reflectance (white, gray, and black, respectively), while in Display 4 . the targets had different reflectances (upper target black, lower target white) but the same luminance. For each display, observers' matches under the condition of monocular viewing (targets appeared coplanar) were compared with those of observers who viewed the array binocularly (targets appeared perpendicular).

Observers. Sixteen naive undergraduates participated, eight under the binocular condition and eight under the monocular condition. The ohservers did not serve as their own controls since, in pilot studies, a set, or experience, effect was discovered. The effect appeared mainly to concern depth perception rather than lightness, however. Thus, the tendency to see the targets as coplanar under monocular viewing seemed to persist into the binocular presentation. Each observer viewed all four displays, although the order was different for each.

Procedure. In the monocular condition, the observer was first asked to look through the aperture and describe the display in general and the spatial position of the target in particular. All eight observers saw the targets as coplanar. The observer's attention was then drawn to the Munsell chart overhead, and the obverver was asked to select a sample from the chart that matched the apparent shade of gray of each of the target surfaces. The observers were unrestricted with respect to time as well as to how often they looked at the target or the chart.

Fssentially the ame procedure was followed for the binocular observers with the exception that the left-hand eyepiece was first adjusted to fit the interocular distance of each observer.

\section{Results and Discussion}

Figure 2 shows the median observer matches. Interobserver reliability was high; the standard deviations for each of the eight target surfaces (both under monocular and binocular conditions) were all less than 1.

Two general observations can be made. First, the luminance relationship between the upper and lower targets was, in each case, seen as a lightness relationship, and second, this was true for both the monocular and binocular conditions. That is, when the targets were seen as perpendicular to one another, 


\begin{tabular}{|c|c|c|c|c|c|c|}
\hline \multicolumn{2}{|c|}{ DISPLAY } & \multirow{2}{*}{ TARGET } & \multirow{2}{*}{$\begin{array}{c}\text { MUNSELL } \\
\text { VALUE }\end{array}$} & \multirow{2}{*}{$\begin{array}{l}\text { LUMINANCE } \\
\text { (FOOTLAMBERTS) }\end{array}$} & \multirow{2}{*}{\multicolumn{2}{|c|}{$\begin{array}{l}\text { MEDIAN OBSERVER MATCH } \\
\text { MONOCULAR BINOCULAR } \\
\end{array}$}} \\
\hline TYPE & NO. & & & & & \\
\hline \multirow{6}{*}{$\begin{array}{l}\text { EQUAL } \\
\text { REFLEC- } \\
\text { TANCE }\end{array}$} & \multirow{2}{*}{1} & UPPER & $\begin{array}{c}9.5 \\
\text { (white) }\end{array}$ & 90.0 & 9.5 & 9.5 \\
\hline & & LOWER & $\begin{array}{c}9.5 \\
\text { (white) }\end{array}$ & 3.0 & 3.25 & 2.75 \\
\hline & \multirow{2}{*}{2} & $\mathbf{u}$ & $\begin{array}{c}5.0 \\
\text { (gray) }\end{array}$ & 23.9 & 9.5 & 9.5 \\
\hline & & L & $\begin{array}{c}5.0 \\
\text { (gray) }\end{array}$ & 0.79 & 2.0 & 2.0 \\
\hline & \multirow{2}{*}{3} & u & $\begin{array}{c}2.0 \\
\text { (black) }\end{array}$ & 3.0 & 5.0 & 4.5 \\
\hline & & L & (black) & 0.1 & 2.0 & 2.0 \\
\hline \multirow{2}{*}{$\begin{array}{l}\text { EQUAL } \\
\text { LUMI- } \\
\text { NANCE }\end{array}$} & \multirow{2}{*}{4} & $\mathbf{u}$ & $\begin{array}{c}2.0 \\
\text { (black) }\end{array}$ & 3.0 & 4.5 & 4.5 \\
\hline & & L & $\begin{array}{l}9.5 \\
\text { (white) }\end{array}$ & 3.0 & 4.5 & 4.5 \\
\hline
\end{tabular}

Figure 2. Matching data for perpendicular planes experiments, Part 1.

they appeared to be of virtually the same lightness as when they were seen as coplanar.

These results are in substantial agreement with previous studies that showed little or no effect of spatial position on lightness, and they appear, at least on the surface, to support the view that perceived lightness is almost entirely governed by ratios of luminance at the retina.

In fact, such a conclusion would be mistaken. To understand this point, it is necessary to be clear about this kind of experiment. What is its essence and what ideas is it capable of testing? These experiments (Displays 1 through 4 ) have the same form as the abovementioned previous studies. In one condition (the monocular condition of these experiments), the target is both retinally adjacent to, and perceived as coplanar with, a second, contextual region. In the other, critical, condition (binocular), the target is again retinally adjacent to the contextual region but is perceptually isolated within its own spatial plane. The first of these conditions does not distinguish among hypotheses; it serves merely as a control. The second condition contains the test: retinal adjacency vs. phenomenal spatial isolation.

This kind of experiment tests the ratio idea against a nonratio idea, such as unconscious registration of illumination. And the results have always shown the importance of ratios. But, given that the ratio approach is fundamentally accepted, it is necessary to pose the kind of question that Rock (Rock \& Brosgole, 1964; Rock \& Ebenholtz, 1962) has so usefully posed in a wide range of studies-namely, should ratios be defined retinally or phenomenally? In other words, is the ratio that determines lightness the ratio between regions that are perceived to lie next to one another in the same spatial plane or is it merely the ratio between adjacent retinal regions? The first of these possibilities will be referred to as the coplanar ratio hypothesis; the second will be termed the retinal ratio hypothesis.

It must be noted that these alternatives cannot be tested by the kind of experiment just reported (Part 1). Consider the binocular condition in these experiments. The retinal ratio hypothesis would clearly predict that the target lightness depends on the luminance ratio between the target and its surrounding region, regardless of where these are perceived to lie in space. But the coplanar ratio hypothesis is unable to make a solid prediction in this case since there is no ratio within the perceived plane of the target, but only the single luminance of the target. The background surface lies at a 45-deg angle to both target surfaces and thus does not provide either target with a coplanar neighbor.

On the other hand, if the display were a bit more complex so that each plane contained multiple luminances, specific predictions could be derived from the coplanar ratio hypothesis. This approach is fundamental to each of the experiments yet to be reported here. Each is a test, in different ways, of the coplanar ratio hypothesis.

\section{PART 2}

\section{Perpendicular Planes, Depth Effect Obtained}

The second group of displays is diagrammed in Figure 3 . The original plan was simply to add a local
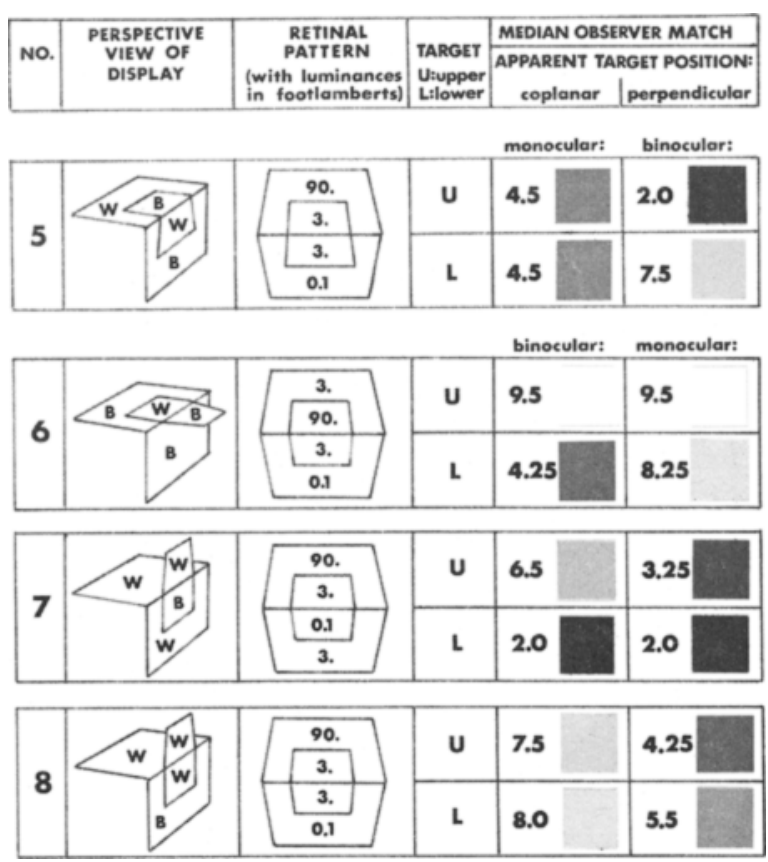

Figure 3. Stimulus displays and matching data for perpendicular planes experiments, Part 2. 
background to each of the targets in the first group of displays. Thus, for example, Display 5 is the logical extension of Display 4. However, this kind of display produced a problem concerning the depth illusion. Notice that, while the lower target in Display 5 is trapezoidal, the lower background is not. The problem arose under monocular viewing when the lower target should have been seen as horizontal (and coplanar with the upper target). In the majority of cases, although not all, the lower target either appeared vertical and coplanar with the lower background or else its spatial position was ambiguous. This is consistent with the known tendency of a surface to be seen in the plane of its background, especially when depth information is weakened, as it is in this case by the elimination of binocular cues.

Therefore, Displays 1, 3, and 4 were extended as shown in Displays 6,7 , and 8 , respectively. (The analogous extension of Display 2 would have required changes in the lighting conditions, so it was omitted.) The difference is that in Displays 1 through 4 (as well as 5), the targets appeared coplanar under monocular viewing and perpendicular under binocular viewing, while in Displays 6, 7, and 8, the targets appeared coplanar under binocular viewing and perpendicular under monocular viewing. Notice that, in these latter displays, the tendency of a surface to be seen in the plane of its background works in favor of the desired depth effects rather than against them. In other words, under monocular viewing when depth information is weak, each target is supposed to appear in the plane of its background. Then, under binocular viewing, the targets easily appear coplanar with each other, as desired.

The inclusion of Display 5 requires some explanation. It was possible to include Display 5 because the above-mentioned problem in creating the appropriate depth appearances was not as serious with this display, possibly owing to the fact that the targets had identical luminances. As it was, additional observers had to be run in order to get a total of eight who saw the spatial positions appropriately. In fact, just over half of the observers did so. Despite these complications, it seemed useful to include Display 5 since it allows interesting comparisons with both Displays 4 and 8 . Display 5 ought to be phenomenally equivalent to Display 8, with the exception that, when the targets appear coplanar, they should appear to lie in the horizontal plane in Display 5 but in the vertical plane in Display 8.

In Displays 5 through 8 , the background squares were $4 \mathrm{in}$. on a side and the targets were 2 -in. squares or the trapezoidal equivalent.

The procedure followed for these displays was identical to that for the first group of displays. As before, separate groups of eight observers participated in the monocular and binocular conditions, respectively, with one exception. A total of $20 \mathrm{ob}$ - servers viewed Display 5 monocularly, but nine of these were excused from the experiment since they failed to see the targets as coplanar. The data reported are from the 11 remaining observers.

\section{Results and Discussion}

In these displays, we find the first substantial effects of apparent spatial position on lightness, even though the array of retinal luminances is again held constant across viewing conditions. Consider the lower target of Display 6 and the upper target of Display 7. The change from apparent coplanarity to apparent perpendicularity of the targets produced changes in perceived lightness of 4 and 3.25 Munsell steps, respectively.

The data of Displays 5 and 8 are troublesome. In these displays, the targets have equal luminances. Thus, they provide a unique situation. Rarely is it the case in our experience that black and white surfaces, situated perpendicularly to each other but sharing a common edge, are differently illuminated by neutral sources just so that a perfect identity of luminance results. Moreover, due to the juxtaposition of the two surfaces, conditions would be optimal for the detection of either a difference in hue or brightness, were there any. Therefore, when the targets are seen as perpendicular, the tendency toward lightness determination by ratios within phenomenal planes is pitted against the stubborn fact of the identity between targets. Consequently, it is not surprising that these displays yielded a much higher variability and that the data tend to be distributed bimodally (when the targets appear perpendicular), some observers seeing the targets as being of the same lightness and some seeing them as black (upper target) and white (lower target).

\section{PART 3}

\section{Retinal Ratios vs. Coplanar Ratios: A Critical Test}

In the experiments just reported, the results differed from what a retinal theory would predict only in a quantitative way. It is possible, however, to construct a critical test in which the coplanar ratio hypothesis would make predictions opposite to those of a retinal theory.

\footnotetext{
Method

Apparatus. The display (shown in Figure 4) consisted of a horizontal, white 4-in. square joined perpendicularly to a vertical, black 4 -in. square. On the left, a black trapezoidal tab extended horizontally from the white square toward the observer. A white trapezoidal tab extended vertically upward from the black square. When viewed monocularly, each tab appeared as a rectangle in the plane of its retinal surround, and these surrounds appeared perpendicular to each other. Viewed binocularly, each surface was seen in its actual position. As hefore, the horizontal surfaces receited 30 times as much light as the vertical, and the luminances of the hlack and white tabs were thus equated.

Giveen naive undergraduates served as observers, eight in the
} 
A

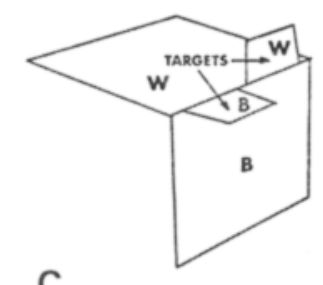

C

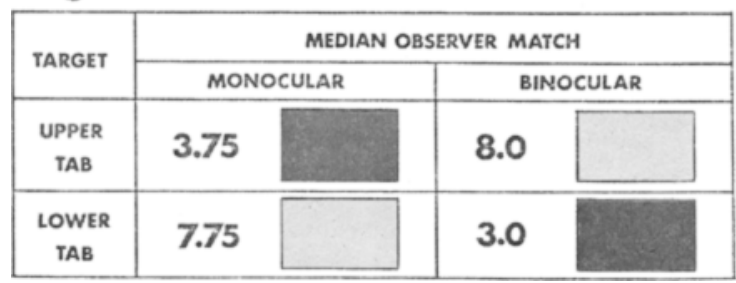

Figure 4. (A) Perspective view of the stimulus display used in the critical test, showing color ( $B$, black; $W$, white) of each part. (B) Monocular retinal pattern showing luminances in footLamberts. (C) Average Munsell matches for monocular and binocular viewing conditions.

monocular and eight in the binocular condition. All saw the appropriate spatial arrangement. The same procedure was followed as in the previous work.

\section{Results and Discussion}

The average Munsell matches are given in Figure 3. Notice that the optic array produced by this display is analogous to the typical simultaneous brightness contrast display. That is, in each case there are two targets of equal luminance, one on a dark background and one on a light background. Retinal theories have always predicted that the target on the light background will appear darker, due to the inhibiting effect of the bright surround. But consider the binocular data in this case. Contrary to retinal predictions, the upper target appeared virtually white, the lower target almost black. Under monocular viewing, these results were reversed, as one would expect from the coplanar ratio hypothesis. That is, the depth illusion caused a switch in the apparent planarity of the tabs. This changed the frame of reference for each tab, which in turn produced a change in the perceived lightness of each tab.

These data do not suggest that the coplanar ratio principle is merely stronger than the retinal principle. In fact, no retinal effect was obtained in this experiment. For instance, if there were a retinal effect (separate from the coplanar ratio effect), it would work in conjunction with the coplanar ratio effect in the monocular condition, but against the coplanar ratio effect in the binocular condition. Thus, we would expect the difference between the upper tab and lower tab matches to be greater in the monocular case than in the binocular case. Yet, this did not occur. Liter- ally the opposite occurred, although the difference was not statistically significant.

These results need to be brought to bear on two important, closely related issues. One is the lightness/brightness distinction; the other is the question of perceived illumination. Regrettably, no data were collected concerning the perceived amount of illumination or the perceived luminance (brightness) of each region. However, since an understanding of the implications of these results is not possible without some conception of how the display appeared in terms of illumination and intensity (as measured by means of a photometer), informal comments of the observers should be considered. First, the observers reported that the two tabs appeared to have similar intensities even though one appeared black and one appeared white. For example, one observer commented that the tabs would have the same intensity in a photograph of the display. Second, observers reported that the horizontal surfaces appeared more brightly illuminated than the vertical surfaces. For a complete description of the targets, one would have to say that one target appeared as a dimly illuminated white, while the other appeared as a brightly illuminated black.

However, although there was complete agreement among observers that the planes appeared differently illuminated, it should be pointed out that these results cannot be explained in terms of the Helmholtzian notion of taking illumination into account. None of the classical cues to illumination, such as penumbra or the sight of the light source, were present.

\section{PART 4 Parallel Planes}

A final experiment was conducted in order to explore the range of application of the coplanar ratio principle. This experiment differed from the previous ones in two ways. First, in this case the planes were parallel but located at different distances from the observer. Second, the depth illusion was produced, not with stereoscopic cues, but by means of false interposition cues.

\section{Method}

Apparatus. To this end, the display illustrated in Figure 5 was created. The observer looked through a pinhole $1 / 16$ in. in diameter, at a dimly lit wall, light gray in color (reflectance $=60 \%$ ) located 56 in. from the pinhole. A 20 -in.-high $\times 91 / 2$-in.-wide doorway-like opening in the wall revealed a second wall twice as far from the pinhole as the first. Attached to the right-hand side of the near wall and extending into the opening were a piece of black paper, $61 / 4$ in. square, and a piece of white paper, $4-5 / 8$ in. high and $4-1 / 8$ in. wide.

The far wall was covered with charcoal-black paper (reflectance $=6 \%$ ) upon which were attached two pieces of paper: a white square, $61 / 4$ in. on a side, and a long gray strip, 3-5/8 in. wide $\times 21^{3 / 4}$ in. high, which partially overlapped the square and ex- 

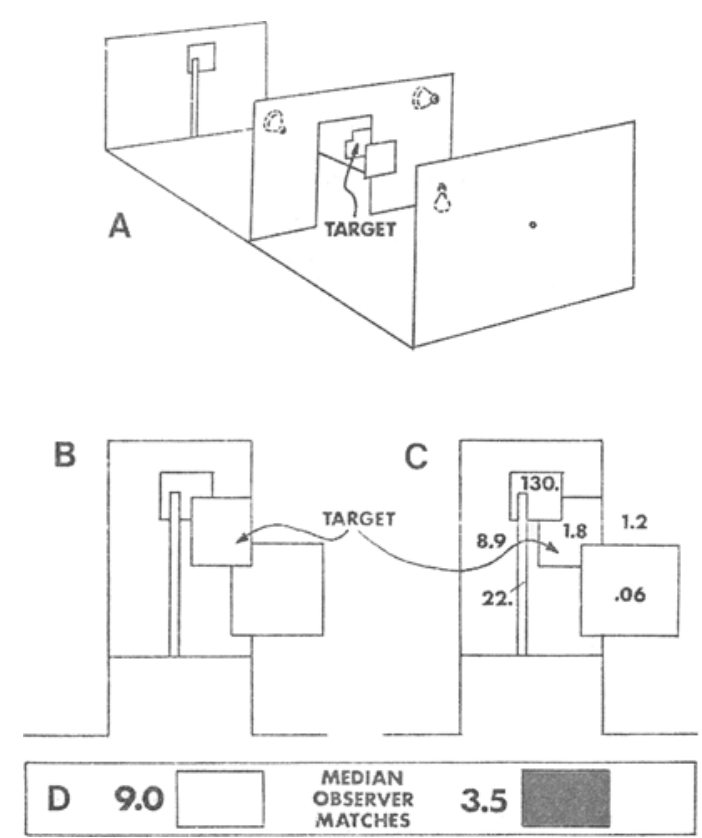

Figure 5. (A) Perspective view of the parallel planes display, showing hidden light bulbs. The display (as seen through the pinhole) in which the target appeared to be located either $(B)$ in the near plane or $(C)$ in the far plane, with luminances shown in foot-Iamberts. (D) The average match from a Munsell chart for the two displays.

tended to the bottom of the wall. The purpose of the gray strip was simply to cause the white square to appear in the far wall, by virtue of interposition, rather than to float in midair in a nearer plane to the observer.

Two 150-W floodlight bulbs, one behind each side of the near wall, illuminated the far wall by 71 times the illumination of the near wall, which was primarily lit by a $25-\mathrm{W}$ light bulb located just behind the pinhole screen. All bulbs were unseen by the observer.

The target surface was the white piece in the plane of the near wall and extending into the doorway. In the experimental condition, its lower right-hand corner was overlapped by a corner of the black square and a $1 \frac{1 / 2}{2} \times 1 \frac{1}{2}$ in. notch was cut out of its upper left-hand corner. As seen through the pinhole, the lower righthand corner of the white square on the far wall coincided with the notch cut out of the target, as in the well-known Ames (1953) demonstrations, such that the distant white square appeared to cover part of the target, causing it also to appear in the plane of the far wall. In the control condition, the target rectangle was complete and overlapped the black square. Thus, it appeared to be in the plane of the near wall, as was objectively the case.

A well-illuminated 16-step Munsell achromatic color chart was located outside the pinhole screen to the lower right of, and facing, the observer.

Observers. Sixteen naive undergraduates participated, eight in the experimental and eight in the control condition.

Procedure. Each observer was asked to look through the pinhole and describe the layout generally and the location of the target specifically. The observer was then asked to select a sample from the Munsell chart that matched the apparent color of the target surface. Observation was monocular for all observers, control and experimental.

\section{Results and Discussion}

These results, shown in Figure 5, provide the strongest support yet for the coplanar ratio principle. The target appeared white in the near condition and almost black in the far condition. It is interesting to note that these results were produced by means of interposition, which is often regarded as a secondary or "cognitive" cue.

\section{GENERAL DISCUSSION}

\section{Differences from Previous Studies}

Why did previous studies fail to find any large effect of perceived spatial arrangement in lightness? The key, as mentioned earlier, lies in the fact that in all of those studies, the target, in one condition, was isolated as the only surface within a plane. The coplanar ratio principle does not tell us directly what will happen in this isolated target condition. What, then, ought we to expect? One theoretical possibility is that the lightness will be indeterminate. We know that under simpler conditions this is true. A single disk of light surrounded by darkness may yield an experience of brightness, but it will not appear as any shade of gray. Indeed, it will not even appear as a reflecting surface at all, but rather as a selfluminous source. Likewise, the lightness (as opposed to brightness) of a ganzfeld is indeterminate.

A second possibility is that the lightness of the isolated target will be determined by its ratio to the noncoplanar context, even though this outcome would not occur with a more complex display. It is this second possibility, what might be called the default outcome, that has been shown to occur empirically. And it is this default outcome that has prevented the observance of large effects of depth on lightness in previous studies. In other words, lightness is always the product of some ratio(s). Therefore, if the lightness of a target is to change from one condition of an experiment to the other, it must be the case that the target is a member of one ratio in one condition, but a different ratio in the other condition. This is often accomplished by changing the intensity of the context. That approach is obviously not acceptable in this case since one is trying to rule out changes in the retinal image. However, the experiments in Parts 2, 3, and 4 illustrate that the requirements can be met, not by changing the intensity of the context, but by providing two possible contexts and manipulating that one of the two that appears to "belong to" the target in a given condition.

The conditions that produced these large spatial position effects differed in two important ways from conditions that have failed to produce such effects. The first, which has just been explained, concerns the need for separate ratios in the two conditions. A second difference, which has not been mentioned, concerns the range of luminances within the display. In each of the displays that produced a spatial position 
effect, the visual system is presented with at least a 900:1 range of luminances. In previous experiments and in Part 1 of this series, the luminance range did not exceed 30:1, a value such that, in all cases, it could have been created with pigments alone, using only a single level of illumination. Thus, it was possible to attribute all luminance differences in the scene to differences in reflectance. Yet, most natural scenes contain a very wide range of luminances, often much greater than even 900:1. Rarely is the range less than $30: 1$. All that is required to exceed $30: 1$ is that, somewhere in the scene, a white surface receive more illumination than some black surface.

The visual system must know, in some sense, that differences greater than 30:1 cannot be created with pigments alone. Thus, when the system is presented with a wide range of luminances either in a natural scene or in the experiments reported here, the incoming pattern of luminances cannot be fitted to an internal representation of the black/white scale without breaking up the range into groups so that the range within any group is no greater than $30: 1$. This is closely related to the idea that differences in luminance within a scene are organized into either differences in reflectance or differences in illumination. This is the point at which planarity comes in; it would make logical sense to group luminances by planes since, in the kind of displays considered here, it is more likely that all the surfaces within a plane are equally illuminated than that parts of separate planes are equally illuminated.

\section{What Does It Mean To Say That Perceived Lightness Depends on Perceived Spatial Arrangement?}

In the earlier report (Gilchrist, 1977) of these findings, I wrote that "If the perceived lightness of surfaces depends on their perceived location in space, depth processing must occur first and be followed by the determination of surface lightness" (p. 187). It now seems to me that this conclusion is unwarranted. A counterexample will illustrate why. Imagine a large sculpture (such as Mt. Rushmore) of a single reflectance. If the sculpture is viewed either at a great distance or through a pinhole, the oculomotor cues are rendered ineffective. Nevertheless, the array is easily seen according to its actual three-dimensional arrangement. This is due to the gradients of illumination produced by the varying slants of the surface. Now these changes in light intensity can only produce the experience of depth if they are correctly seen as changes in illumination rather than as changes in surface lightness. Were they seen as changes in surface lightness, the entire sculpture would appear flat. Therefore, if the three-dimensional layout is correctly perceived, this already implies that a decision about the lightness (uniform) of the sculpture has been made. Either the array will be seen as (1) being flat, uniform in illumination, and with varying shades of gray, or else as (2) varying in depth, varying in illumination, and with a uniform shade of gray. The point is that, in these cases, a decision about lightness is the same as a decision about depth.

Bergstrom (1977) has reached similar conclusions using an analysis of the kind Johansson has used for motion. Bergstrom presented observers with a pattern of luminance gradients that might be described as a decreasing series of luminance steps, although the step changes were gradual, not sharp. The predominant perception was of a corrugated surface (much like roofing tiles) of uniform reflectance, illuminated from one side. Bergstrom proposes that the interrelated perceptions of reflectance, illumination, and three-dimensional shape all emerge simultaneously from an analysis of the retinal image. In his example, as in the sculpture example, it is not appropriate to ask whether depth perception or lightness perception occurs first.

Even if depth perception does not precede lightness as a general rule, it is still useful to ask whether depth precedes lightness in the experiments reported here. Under these conditions, one can vary perceived depth while holding the retinal image constant (or nearly so) and produce changes in perceived lightness. Notice that this could not be done in the Mt. Rushmore or Bergstrom cases since there is no independent source of depth information in those cases. There, the source of depth information is the same as the source of lightness and illumination informationnamely, the retinal luminance gradients.

From this, we can conclude that lightness depends on perceived depth in the present experiments more truly than in the Bergstrom case. In that case, it makes as much sense to say that depth depends on lightness as to say that lightness depends on depth. But, in the present experiments, there is an asymmetry, owing to the presence of a partially independent source of depth information. Nevertheless, the dependence of lightness on depth in these experiments does not necessarily establish the fact that depth perception precedes lightness perception in time, even under these conditions.

To summarize, we may say that the expression "depends on" can have at least three separate meanings: (1) is preceded by, (2) is caused by, and (3) is intimately related to. The first of these meanings must be rejected, certainly in terms of a general principle. Even under conditions such as those of the present experiments, in which perceived depth could logically precede perceived lightness, this has not yet been proven. The second meaning is ambiguous since it implies an outmoded concept of causality involving a linear sequence of discrete stages, as in the billiard ball metaphor. This conception is too crude to be of 
much use in describing the extraordinary degree of interdependence among perceived qualities. The third of these meanings seems to be the most appropriate. Whatever the exact relationship between perceived lightness and perceived depth, it should be clear by now that these perceptual qualities cannot be understood in isolation from each other.

\section{Percept-Percept Relationships}

The evidence presented here is consistent with a by-now substantial body of findings indicating that perceived variables, such as perceived depth, have a strong effect on phenomena often thought to be determined solely by proximal stimulus variables. For instance, Lehmkuhle and Fox (1980) have recently shown that masking effects are much stronger when the target and induction figures are perceived to lie in the same plane. White (1976) had earlier shown that optimal masking effects occur when the target and induction figures are perceived as located in adjacent spatial positions rather than when they are retinally adjacent. Although retinal adjacency and perceived adjacency (in the frontal plane) are normally confounded, White teased them apart using a moving-eye technique analogous to that introduced by Rock and Ebenholtz (1962) to demonstrate that stroboscopic motion requires a change of perceived location, not a change of retinal location. The crucial role of perceived depth in stroboscopic motion has been shown by Attneave and Block (1973) and Corbin (1942), and its role in induced motion has been shown by Gogel and Koslow (1972).

These percept-percept relationships raise many important, yet difficult, issues for perceptual theory. Although a full analysis of these issues will not be attempted here, a few relatively simple points can be made. These findings underscore the problems with theories that propose direct, one-to-one relationships between stimulus variables and perceptual variables. Particularly vulnerable here is the assumption, implicit in much sensory physiological work, that perception is accomplished by a vast collection of detector mechanisms.

The same results were produced in the present research using stereoscopic cues in one case and interposition cues in another. It is likely that these results would be produced by any method that created the same "perceived spatial arrangement." Thus, the prospects seem remote for a successful theory that does not involve processes central to the very intelligence of the perceiving organism.

When higher order variables are included as stimulus variables, as in Gibson's (1966) approach, the case is not so simple, although a number of writers (Epstein, 1977; Gogel, 1973a, 1973b; Hochberg, 1974) have argued that percept-percept dependencies provide a fundamental challenge to Gibson's generalized psycho- physical hypothesis. Despite the undeniable importance of ecological optics, and despite the untold potential of higher order variables, it seems doubtful that a pure stimulus theory will ever provide a complete account of perception, if organismic factors are ignored.

These percept-percept dependencies seem to require an approach in which seeing and thinking are closely related. As such an approach, Rock's (1975, 1977) unconscious inference theory appears to receive the most direct support from these findings. Although the detailed workings of this system have yet to be made explicit, the concept of the output of one process serving as the input of another seems consistent with an inferential system. Certainly, sensory stimulation would have to be processed in a logical way if perception is to be at all veridical.

Another theoretical approach that could, in principle, assimilate these findings is that of Johansson (1970, 1974, 1977). Although Johansson has not dealt explicitly with the issues raised by perceptpercept relationships, his approach does seem to combine the necessary ingredients, which include (1) an emphasis on ecological optics and recognition of the importance of higher order variables, (2) an acknowledgment of the role of organizing principles inherent in the organism, and (3) a view of perception as logical and closely related to higher forms of thought.

To the extent that these findings demonstrate the extraordinary interdependence of perceptual variables, they support an important theme of Gestalt psychology. A theory of the perception of green, with no reference to the perception of other colors, would seem artificial, atomistic, and absurd. Yet, it has been widely believed that a theory of lightness perception need make no reference to perceived depth (or to perceived illumination). The atomism of this belief is suggested by the intimate way that illumination, reflectance, and spatial position interact to produce the gradients of stimulation that fuel the visual system.

\section{Coplanar Ratios and Gogel's Adjacency Principle}

These results may be regarded as supporting Gogel's adjacency principle (1973a, 1973b). The parallel planes experiment is very similar to the study reported by Gogel and Mershon (1969) and another by Mershon (1972). The situation is not so clear, however, in the case of the perpendicular planes experiments. The issue here is one of planarity vs. adjacency. Two surfaces that meet at a corner are not coplanar. They are adjacent, however, unless the adjacency principle is somehow understood to include closeness in orientation, and the perpendicular planes experiments show that lightness is not determined by the luminance ratio between two such perpendicularly positioned surfaces, even if they are adjacent along a common edge. 


\section{Laboratory Data vs. Experience}

The results obtained in this work will no doubt be regarded by many as surprising. And yet, they might have been predicted if certain implications of the retinal theories of lightness perception had been closely examined. For example, according to the retinal theories, a patch of light will appear as a darker shade of gray if it is situated next to a brighter patch in the retinal image. But this implies that when surfaces meeting at a corner are differentially illuminated (as is virtually always the case), the shadowed side must be subject to a major lightness illusion. Yet, this is generally not the case. Now the shadowed side of a corner sometimes appears slightly darker in surface lightness than it is, and perhaps this explains why this incongruity between fact and theoretical prediction has gone unnoticed. But the magnitude of the loss of constancy is critical here. It is not unusual for one side of a white house, for instance, to receive 30 times as much illumination as an adjacent, shadowed side. Thus, according to retinal theories, the shadowed side ought to appear apyroximately black, not merely "slightly darker" than the other wall."

Consider another example. If the inside frame of a window through which I am looking receives more light than the scene viewed through the window, surfaces in the scene should appear much darker in color than they actually are. Instead, two things are apt to happen. First, the outside scene may appear more dimly illuminated than it actually is, a kind of illumination contrast. Second, and more important, the surfaces in the outside scene will become harder to see. But, it is one thing when it gets difficult to see what color a surface is, and it is quite another thing for the surface to appear darker in color. One suspects that considerations such as these have been neglected due, in part, to a certain embarrassment with introspection and the subsequent pressure to base theories firmly on laboratory data. But the need for dependable empirical data ought not lead us to deny obvious facts of perception out of theoretical necessity, even if those theories are derived from laboratory data.

\section{Theories of Lightness Perception}

The results contain serious implications for virtually all the major theories of lightness perception. The most direct challenge is to those theories that place great emphasis on the retinal pattern. In particular, these results raise serious doubts about the adequacy of models that regard lateral inhibition of retinal elements as the fundamental mechanism responsible for lightness constancy. One of the most direct implications of the lateral inhibition explanation is that if there is no change in the retinal image, there can be no change in perceived lightness. Yet, in these experiments, essentially identical retinal patterns were used to produce the appearance of white in one case and black in another, almost a change from one end of the lightness scale to the other!

Cornsweet's (1970) model, tied as it is to the retina and to lateral inhibition, would appear to be particularly vulnerable here. The opponent-process theory of Jameson and Hurvich (1964) would appear to fall into the same category, although the matter is not quite as simple in this case. Jameson and Hurvich make it clear that "the locus of the opponent induction effects is by no means limited to the level of retinal photochemistry" (1964, p. 139). However, although they do not necessarily place the "mechanism" of the opponent response at the retina, nevertheless, the response is held to be governed, in their model, by relative intensities of adjacent parts of the retinal image.

The previous statement applies equally well to Wallach's ratio theory. However, Wallach's conception could account for the data presented here if the idea of "retinal ratio" were replaced with the idea of "apparent coplanar ratio."

Helson's (1943) adaptation-level theory has received broad application in many areas of psychology, in addition to being a major theory of lightness perception. The question is, would the adaptation level be held to change, given virtually no change in the retinal image? It seems unlikely that it would or that these results would have been predicted by adaptation-level theory.

The theories of Cornsweet, Helson, Jameson and Hurvich, and Wallach all share a crucial common feature. All hold that perceived lightness can be explained without reference to perceived illumination. To understand this position, it must be realized that these theories were all developed in reaction to the classic position of Helmholtz, who believed that lightness was determined by unconsciously taking into account the level of illumination. It is not surprising that this theory has been widely rejected. Empirical results have failed to prove the effectiveness of those cues that would seem to be necessary to determine the level of illumination. This can be seen in the present study as well. Although, as mentioned earlier, the separate planes (in Parts 2, 3, and 4) did appear to be differentially illuminated, cues such as penumbra and sight of the light source were completely absent from the display. Thus, these results do not support a return to the Helmholtzian formula.

Nevertheless, it seems rash to argue that lightness perception can be explained with no reference to perceived illumination, since the intensity dimension of retinal stimulation must mediate our perception of both lightness and illumination. The only way to avoid this conclusion is to deny that illumination is perceived. And this seems to be precisely the position, whether implicit or explicit, of the retinal theories. ${ }^{2}$ 
That is, the intensity mechanism of the visual system is reserved exclusively for the perception of surface qualities. Yet, this limitation flies in the face of experience. How can it be denied that we perceive illumination? We see shadows, both cast and attached. We see increments of illumination, such as the light provided by a spotlight. And, ultimately, our experience of surfaces is dual; every surface is perceived at a certain lightness value as well as having a particular value of illumination. The important point is that one can accept the idea of illumination perception, even the idea that it is intimately linked to lightness perception, without accepting the Helmholtzian formula.

\section{The Role of Lateral Inhibition}

These empirical results seriously question the possibility of explaining lightness constancy by lateral inhibition. But this possibility can be questioned on logical grounds alone.

Cornsweet (1970, p. 365) has offered an explanation of how the contrast function of lateral inhibition could work to produce lightness constancy. A key feature of this explanation is that differences in the rates of firing of cells corresponding to adjacent (but unequal in luminance) regions of the retinal image are enhanced through lateral inhibition. But it has not been generally recognized that such an enhancement of edge differences could work in favor of constancy only in some cases-that is, at some edges. Herein lies the Achille's heel of the contrast approach. Theories such as those of Cornsweet and of Jameson and Hurvich have failed to make a critical distinction concerning edges (or ratios). Fundamentally, these are of two types: edges that are produced by, and perceived as, changes in reflectance, and edges that are produced by, and perceived as, changes in illumination. These contrast theories have implicitly treated all edges as reflectance edges. Furthermore, the theories have typically only been evaluated using displays that consisted totally of reflectance edges. But it can be shown that the theories make predictions that go in the wrong direction when applied to illumination edges.

For instance, consider a shadow cast on a white wall. Normally, this is perceived as a shadow, not as a dark gray surface. Yet, to be consistent, a lateral inhibition model must assume that contrast would operate at the border of the shadow ${ }^{3}$ to increase the apparent difference between the shadow and its background wall. But this would work against constancy, which in this case would require the shadowed/ nonshadowed difference to be disregarded, if the wall is to be seen as uniformly white.

A great deal of recent work, especially work on stabilized retinal images, has suggested that the retinal image is best thought of as an array of edges, rather than an array of patches of light. The next logical step is to realize that some of these edges are illumination edges and others are reflectance edges. Therefore, the constancy problem, which fundamentally deals with how the effects of refiectance are separated from the effects of illumination, would seem to require a means of disentangling and sorting out these two basic kinds of edges. A contrast mechanism, such as lateral inhibition, that must be applied indiscriminately to all the edges in the image is simply unable to come to grips with the basic problem.

By failing to make the distinction between illumination and reflectance edges, the contrast theories imply that all the edges in a scene are reflectance edges. If this were true, if all edges were reflectance edges, there would be no constancy problem, and lightness perception would be a fairly simple matter. As Rock (1975) has pointed out, "Were it not for variations in illumination, there would be a high correlation between the intensity of light reflected to the eye by a surface and the perceived neutral color of that surface" (p. 502). Therefore, if the contrast theories can only deal with the case of a scene composed entirely of reflectance edges, they are in the embarrassing position of explaining just that aspect of lightness constancy that needs little or no explanation.

Thus, it would appear that lateral inhibition should not be expected to account for such a complex problem as lightness constancy. A more reasonable conception would be that lateral inhibition plays an important role in setting up the kind of edge signal that has been shown by Krauskopf (1963), Walraven (1976), Whittle (1969), and Yarbus (1967) to be so effective in color perception. Therefore, in one sense, lateral inhibition can be likened to opening one's eyes; both are necessary for perception, but neither explains lightness constancy.

\section{REFERENCES}

Ames, A., JR. Reconsideration of the origin and nature of perception in situations involving only inorganic phenomena. In S. Ratner (Ed.), Vision and action. New Brunswick, N.J: Rutgers University Press, 1953.

Attneave, F., \& Block, G. Apparent movement in tridimensional space. Perception \& Psychophysics, 1973, 13, 301-307.

BEck, J. Apparent spatial position and the perception of lightness. Journal of Experimental Psychology, 1965, 69, 170-179.

Bergstrom, S. S. Common and relative components of reflected light as information about the illumination, colour, and threedimensional form of objects. Scandinavian Journal of Psychology, 1977, 18, 180-186.

Corbin, H. H. The perception of grouping and apparent movement in visual depth. Archives of Psychology, 1942, No. 273.

Connsweet, T. Visual perception. New York: Academic Press, 1970.

Epstrin, W. Phenomenal orientation and perceived achromatic color, Journal of Psychology, 1961, 52, 51-53.

Epsterin, W. What are the prospects for a higher-order stimulus theory of perception? Scandinavian Journal of Psychology, $1977,18,164-171$. 
Flock, H. R., \& Freedberg, E. Perceived angle of incidence and achromatic surface color. Perception \& Psychophysics, $1970,8,251-256$.

GibBs, T., \& Lawson, R. B. Simultaneous brightness contrast in stereoscopic space. Vision Research, 1974, 14, 983-987.

GiBson, J. J. The senses considered as perceptual systems. New York: Houghton Mifflin, 1966.

Gilchrist, A. L. Perceived lightness depends on perceived spatial arrangement. Science, 1977, 195, 185-187.

Goge L, W. C. The organization of perceived space. 1. Perceptual interactions. Psychologische Forschung, 1973, 36, 195-221. (a)

Gogel, W. C. The organization of perceived space. II. Consequences of perceptual interactions. Psychologische Forschung, 1973, 36, 223-247. (b)

Gogel, W, C., \& KosLow, M. The adjacency principle and induced movement. Perception \& Psychophysics, 1972, 11, 309-314.

Gogel, W. C., \& Mershon, D. H. Depth adjacency in simultaneous contrast. Perception \& Psychophysics, 1969, 5, 13-17.

HeLmholTz, H. von. [Helmholtz's treatise on physiological optics] (3rd German ed.; J. P. Southall, Ed. and trans.). New York: Dover, 1962. (Originally published, 1867.)

Helson, H. Some factors and implications of color constancy. Journal of the Optical Society of America, 1943, 33, 555-567.

HERING, E. [Outlines of a theory of the light sense] (L. M. Hurvich \& D. Jameson, trans.). Cambridge, Mass: Harvard University Press, 1964. (Originally published, 1874.)

HochBe RG, J. Higher-order stimuli and inter-response coupling in the perception of the visual world. In R. B. MacLeod \& H. L. Pick, Jr. (Eds.), Perception: Essays in honor of James J. Gibson. Ithaca, N. Y: Cornell University Press, 1974.

Hochвerg, J. E., \& Beck, J. Apparent spatial arrangement and perceived brightness. Journal of Experimental Psychology, $1954,47,263-266$.

JAmeson, D., \& Hurvich, L. M. Theory of brightness and color contrast in human vision. Vision Research, 1964, 4, 135-154.

Johansson, G. On theories for visual space perception: A letter to Gibson. Scandinavian Journal of Psychology, 1970, 11, 67-74.

Johansson, G. Projective transformations as determining visual space perception. In R. B. MacLeod \& H. L. Pick, Jr. (Eds.), Perception: Essays in honor of James J. Gibson. Ithaca, N.Y: Cornell University Press, 1974.

Johansson, G. Spatial constancy and motion in visual perception. In W. Epstein (Ed.), Stability and constancy in visual perception: Mechanisms and processes. New York: Wiley, 1977.

Julesz, B. Foundations of Cyclopean perception. Chicago: University of Chicago Press, 1971.

KRAUSKOPF, J. Effect of retinal image stabilization on the appearance of heterochromatic targets. Journal of the Optical Society of America, 1963, 53, 741-744.

LEнмкuнle, S., \& Fox, R. Effect of depth separation in metacontrast masking. Journal of Experimental Psychology: Human Perception and Performance, 1980, 6, 605-621.
Mershon, D. H. Relative contributions of depth and directional adjacency to simultaneous whiteness contrast. Vision Research, 1972, 12, 969-979.

Rock, I. An introduction to perception. New York: Macmillan, 1975.

Rock, I. In defense of unconscious inference. In W. Epstein (Ed.), Stability and constancy in visual perception: Mechanism and processes. New York: Wiley, 1977.

Rock, I., \& Brosgole, L. Grouping based on phenomenal proximity. Journal of Experimental Psychology, 1964, 67, 531-538.

Rock, I., \& Ebenholtz, S. Stroboscopic movement based on change of phenomenal rather than retinal location. American Journal of Psychology, 1962, 75, 193-207.

WALLACH, H. Brightness constancy and the nature of achromatic colors. Journal of Experimental Psychology, 1948, 38, 310-324.

Walraven, J. Discounting the background: The missing link in the explanation of chromatic induction. Vision Research, 1976, 16, 289-296.

Whiтe, C. W. Visual masking during pursuit movements. Journal of Experimental Psychology: Human Perception and Performance, 1976, 2, 469-478.

Whittle, P., \& Challands, P. D. C. The effect of background luminance on the brightness of flashes. Vision Research, 1969 , 9, $1095-1110$.

Yarbus, A. L. Eye movements and vision. New York: Plenum Press, 1967.

\section{NOTES}

1. When there is a 30:1 ratio between adjacent surfaces that appear in the same plane, the darker side appears black.

2. For example, Cornsweet $(1970$, p. 380) states that "our perceptions are correlated with a property of objects themselves (i.e., their reflectances) rather than with the incident illumination."

3. It has been claimed that there would be less contrast at the border of shadows since such borders are often gradual changes in luminance, rather than stepwise. However, natural scenes are replete with sharp illumination borders. The borders of attached shadows are sharp if the change in planarity (corner) of the surface is sharp. Even cast shadows have sharp borders if (a) the illumination is from a point source, (b) the shadow-casting object is close to the projection surface, or (c) the distance between the observer and the shadow is great (although the actual shadow border may not be sharp, the retinal image of the border gets sharper with increases in viewing distance).

(Received for publication November 30, 1979; revision accepted June 30,1980 .) 